Revista de

Estudios

Kantianos

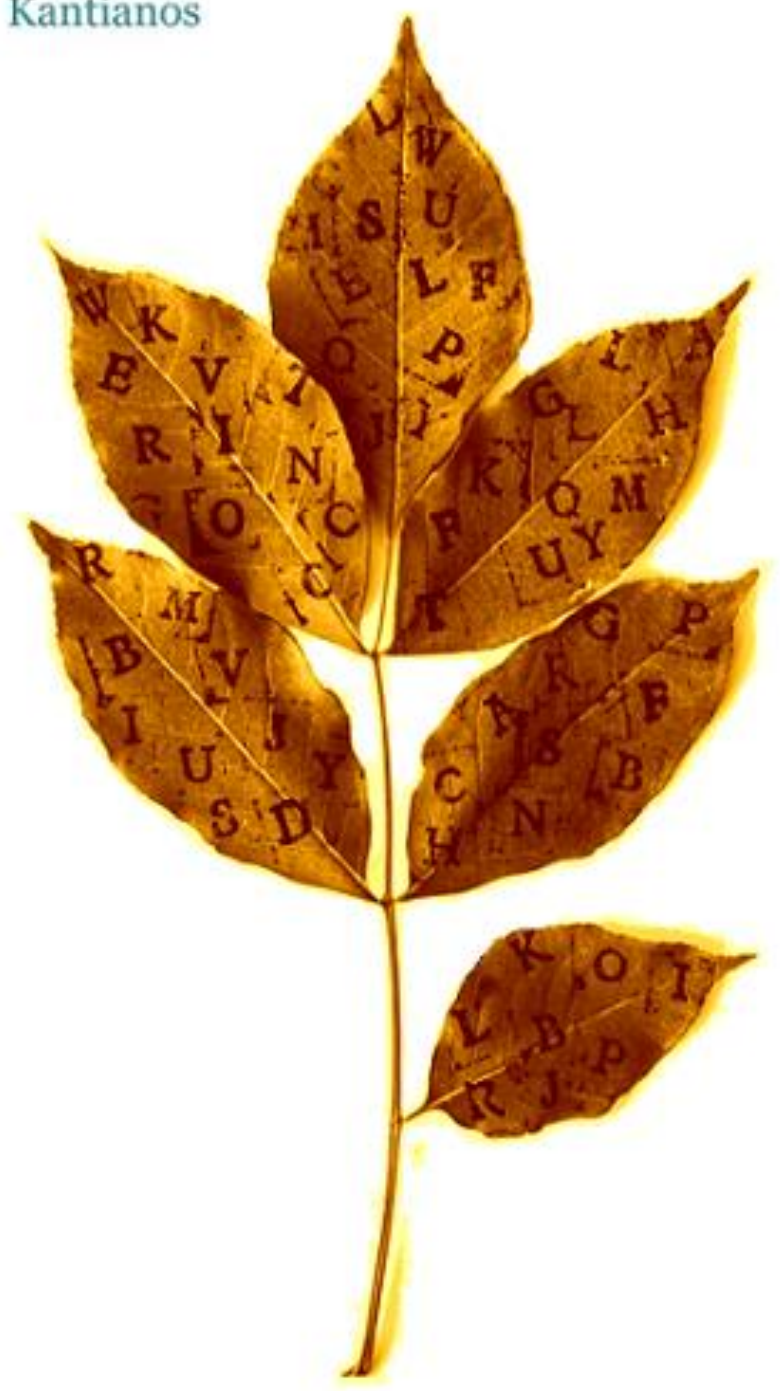




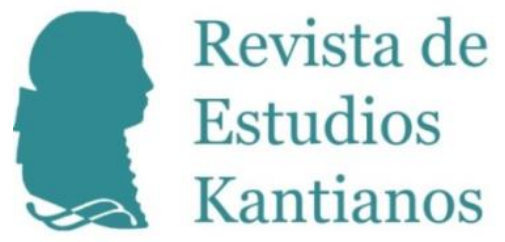




\title{
Revista de Estudios Kantianos
}

\author{
Publicación internacional de la Sociedad de Estudios Kantianos en Lengua Española \\ Internationale Zeitschrift der Gesellschaft für Kant-Studien in Spanischer Sprache \\ International Journal of the Society of Kantian Studies in the Spanish Language
}

\section{Dirección}

Fernando Moledo, FernUniversität in Hagen

fernando.moledo@fernuni-hagen.de

Hernán Pringe, CONICET-Universidad de Buenos Aires/ Universidad Diego Portales, Santiago de Chile

hpringe@gmail.com

\section{Secretario de edición}

Óscar Cubo Ugarte, Universitat de València

oscar.cubo@uv.es

\section{Secretaria de calidad}

Alba Jiménez Rodríguez, Universidad Complutense de Madrid albjim04@ucm.es

\section{Editores científicos}

Jacinto Rivera de Rosales, UNED, Madrid

Claudia Jáuregui, Universidad de Buenos Aires

Vicente Durán, Pontificia Universidad Javeriana, Bogotá

Julio del Valle, Pontificia Universidad Católica del Perú, Lima

Jesús Conill, Universitat de València

Gustavo Leyva, Universidad Autónoma de México, México D. F.

María Xesús Vázquez Lobeiras, Universidade de Santiago de Compostela

Wilson Herrera, Universidad del Rosario, Bogotá

Pablo Oyarzun, Universidad de Chile, Santiago de Chile

Paula Órdenes Azúa, Universität Heidelberg 


\section{Comité científico}

Juan Arana, Universidad de Sevilla

Reinhardt Brandt, Philipps-Universität Marburg

Mario Caimi, Universidad de Buenos Aires

Monique Castillo, Université de Paris-Est

Adela Cortina, Universitat de València

Bernd Dörflinger, Universität Trier

Norbert Fischer, Universität Eichstätt-Ingolstadt

Miguel Giusti, Pontificia Universidad Católica del Perú

Dulce María Granja, Universidad Nacional Autónoma de México

Christian Hamm, Universidad Federal de Santa María, Brasil

Dietmar Heidemann, Université du Luxembourg

Otfried Höffe, Universität Tübingen

Claudio La Rocca, Università degli Studi di Genova

Juan Manuel Navarro Cordón, Universidad Complutense, Madrid

Carlos Pereda, Universidad Nacional Autónoma de México

Gustavo Pereira, Universidad de la República, Uruguay

Ubirajara Rancan de Azevedo, Universidade Estadual Paulista, Brasil

Margit Ruffing, Johannes Gutenberg-Universität Mainz

Gustavo Sarmiento, Universidad Simón Bolívar, Venezuela

Sergio Sevilla, Universitat de València

Roberto Torretti, Universidad Diego Portales, Santiago de Chile

Violetta Waibel, Universität Wien

Howard Williams, University of Aberystwyth

Allen W. Wood, Indiana University

Diseño, revisión de estilo, corrector y maqueta

Josefa Ros Velasco, Harvard University, Cambridge (MA)

\section{Entidades colaboradoras}

Sociedad de Estudios Kantianos en Lengua Española (SEKLE)

Departament de Filosofia de la Universitat de València

Instituto de Humanidades, Universidad Diego Portales

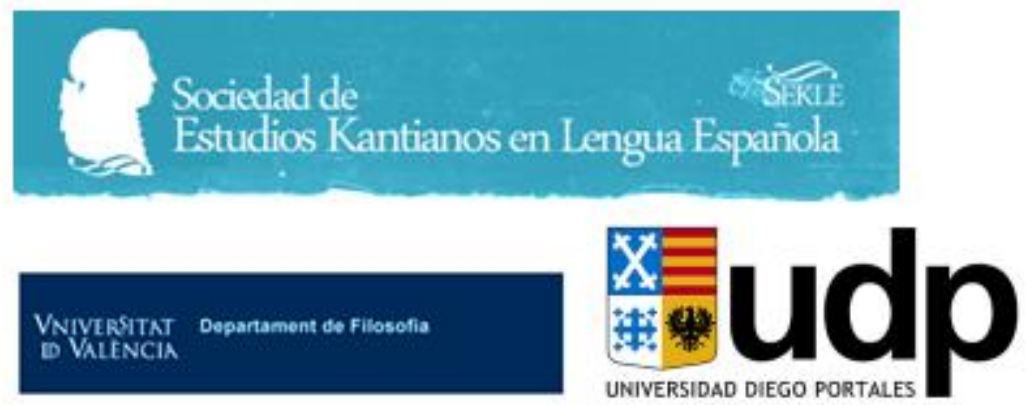




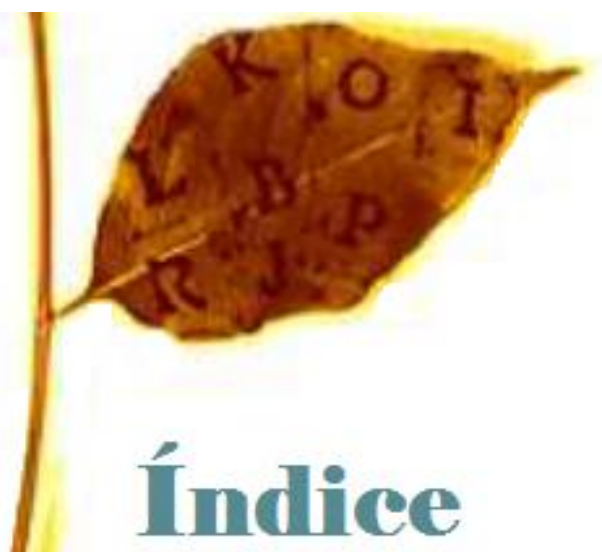

\section{Artículos}

193 Phänomenologie oder Kritizismus? Zur Ausenandersetzung zwischen Eugen Fink und

Rudolf Zocher

Christian Krijnen

DOI 10.7203/REK.4.2.13750

221 Una interpretación de la Stufenleiter de A320/B376. Contribución a la determinación precisa del carácter de la distinción entre intuiciones y conceptos

Luis Placencia

DOI 10.7203/REK.4.2.15537

\section{La actualidad de la Crítica de la razón pura: Parte Teórica}

245 Presentación de los editores al número monográfico. La actualidad de la Crítica de la razón pura: Parte Teórica

David Hereza; Óscar Cubo

DOI 10.7203/REK.4.2.15571

249 "Los primeros pensamientos de Copérnico" $(\mathrm{KrV}, \mathrm{Bxvi})$

Gonzalo Serrano Escallón

DOI 10.7203/REK.4.2.13930

270 El espacio en cuanto forma de los fenómenos y la tesis de la receptividad: mutua implicación

Diana Gloria Contreras Gallegos

DOI 10.7203/REK.4.2.14005

293 La idealidad del tiempo, Gödel y la relatividad

Gilberto Castrejón

DOI 10.7203/REK.4.2.13825 
319 Synthetische und analytische Einheit der Apperzeption. Über ein nach wie vor aktuelles und missverständliches Problem der „Kritik der reinen Vernunft“

Carsten Olk

DOI 10.7203/REK.4.2.13784

338 Una defensa de la actualidad de la Crítica de la razón pura

Alejandro Lanchas Sánchez.

DOI 10.7203/REK.4.2.13840

352 Substancia, cambio y materia en las Analogías de la experiencia de la Crítica de la razón pura de Kant

Jacinto Rivera de Rosales

DOI 10.7203/REK.4.2.15752

382 La refutación kantiana del idealismo y el realismo ingenuo reconsiderado

Manuel Amado

DOI 10.7203/REK.4.2.13963

397 La concepción kantiana de la verdad: Entre la correspondencia y la argumentación Ana María Andaluz Romanillos

DOI 10.7203/REK.4.2.14094

423 Die konzeptualistische und die nicht-konzeptualistische Interpretation der

Kooperationsthese

Sophia Maddalena Fazio

DOI 10.7203/REK.4.2.13996

434 Geist im Sinnlichen. Eine Deutung der transzendentalen Ideen im Ausgang von Kants

Anthropologie

Margit Ruffing

DOI 10.7203/REK.4.2.14336

452 La deducción metafísica de las ideas a partir de las formas del silogismo

Mario Pedro Miguel Caimi

DOI 10.7203/REK.4.2.14015

476 The epistemological interpretation of transcendental idealism and its unavoidable slide into compatibilism

Daniel Dal Monte

DOI 10.7203/REK.4.2.13939

508 Estructura argumentativa, unidad temática y coherencia doctrinal en los Progresos de la metafísica de Immanuel Kant

Marcos Thisted

DOI 10.7203/REK.4.2.14102

525 Sentido y límites de la filosofía transcendental en el proyecto kantiano

Salvi Turró

DOI 10.7203/REK.4.2.13919 


\section{Recensiones}

546 Gualtiero Lorini y Robert B. Louden (Eds.): Knowledge, Morals and Practice in Kant's Anthropology. Nueva York, Palgrave Macmillan, 2018, 171 pp. ISBN: 978-3-319-98726-2 Natalia Andrea Lerussi DOI 10.7203/REK.4.2.15773

552 Paula Órdenes y Anna Pickhan (Eds.): Teleologische Reflexion in Kants Philosophie. Weisbaden, Springer, 2019, 310 pp. ISBN: 978-3-658-23693-9

Rafael Reyna Fortes

DOI 10.7203/REK.4.2.15851

\section{Traducción}

556 Los Aforismos sobre religión y deísmo de J. G. Fichte. Presentación, debates abiertos y traducción

María Jimena Solé

DOI 10.7203/REK.4.2.15772

\section{Eventos y normas para autores}

583 Normas para autores

DOI 10.7203/REK.4.2.15906 


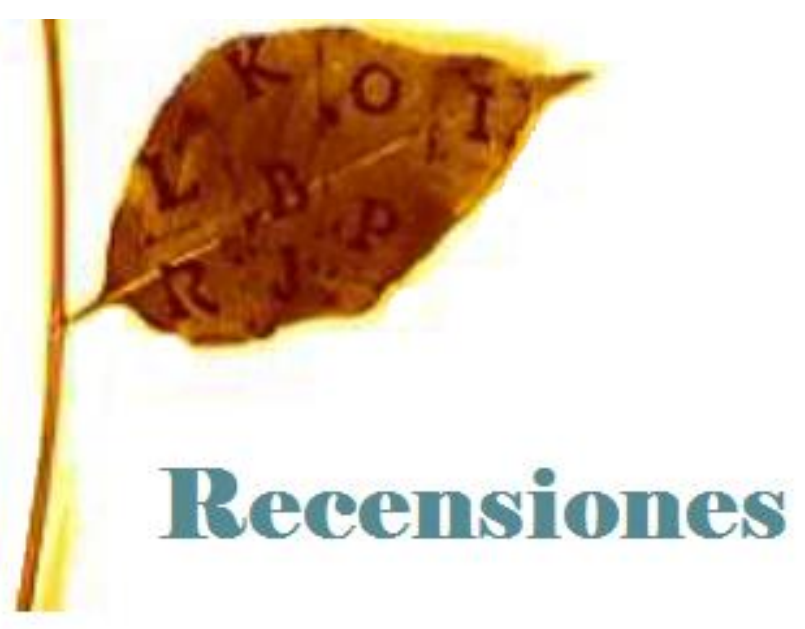




\title{
Gualtiero Lorini y Robert B. Louden (Eds.): Knowledge, Morals and Practice in Kant's Anthropology. Nueva York, Palgrave Macmillan, 2018, 171 pp. ISBN: 978-3-319-98726-2
}

\author{
NATALIA ANDREA LERUSSI
}

Knowledge, Morals and Practice in Kant's Anthropology es un libro editado recientemente por Gualtiero Lorini y Robert B. Louden sobre la antropología de Immanuel Kant. El libro consta de una introducción a cargo de los editores y nueve artículos (de reconocidos especialistas en el área), subdivididos en dos partes: i) Sources and Influences in Kant's Definition of the Knowledge Concerning the Human Being y ii) The Peculiarities of Anthropological Knowledge in Kant: Metaphysics, Morals, Psychology, Politics. El libro abre con un resumen de los antecedentes académicos de quienes escriben, seguido por una sección que informa del modo como se citan las diferentes obras de Kant, las ediciones y las traducciones adoptadas en el libro, y cierra con un útil índice de nombres y palabras. Se trata de una edición cuidadosa y cómoda para el lector.

Como indican los editores en la "Introducción" el objetivo del libro es desarrollar algunas tendencias adoptadas recientemente en la lectura de la antropología de Kant, así como ofrecer algunas innovaciones. Particularmente, buscan definir la especificidad y el aporte del sendero que abre la antropología de Kant; alternativo y complementario, según los editores, a la vía de la filosofía trascendental. Las tendencias remiten, por un lado, a las investigaciones que dan cuenta de esta disciplina desde las fuentes de la ilustración alemana y Rousseau y, por otro, a las lecturas 'internas' que dan cuenta de la originalidad del aporte de Kant y que inscriben su antropología dentro del gran proyecto crítico. En las páginas 2-3 los editores defienden la tesis según la cual la filosofía trascendental y la antropología se ocupan ambas, esencialmente, del 'sujeto humano'; la primera desde un punto de vista a priori y la segunda desde una perspectiva a posteriori. De ahí que

1 CONICET - Universidad de Buenos Aires. Contacto: natalialerussi@gmail.com. 
sostengan la relevancia que tiene la incorporación de los aportes de la antropología kantiana en cualquier comprensión adecuada de su filosofía trascendental. Esta tesis es muy importante. Ciertas lecturas canonizadas de la filosofía kantiana se han tomado demasiado en serio las pretensiones de Kant acerca de que lo trascendental está separado por un abismo de lo empírico y han desconsiderado, en consecuencia, solamente las fuentes que hablan a favor de lo contrario. Este libro considera, seriamente, aquellas otras fuentes y pone a la luz elementos que no pueden desconsiderarse si se pretende comprender la filosofía de Kant: el laboratorio desde el cual emergió, las tradiciones de las cuales Kant bebió, etc. Creo que las diferentes propuestas del libro colaboran a renovar y oxigenar el legado del filósofo.

En la primera parte, Sources and Influences in Kant's Definition of the Knowledge Concerning the Human Being, seis especialistas de la filosofía kantiana (Wilson, Favaretti Camposampiero, Goubet, Lorini y Lyssy) estudian la presencia, fundamentalmente, de Thomasius, Wolff, Crusius, Baumgarten, Meier, Lambert y Rousseau en la antropología de Kant.

En "Elucidations of the Sources of Kant's Anthropology" Holly Wilson introduce el debate acerca de las fuentes principales de las lecciones de antropología de Kant. Fundamentalmente, discute las tesis de Adickes y Brandt que enfatizan el vínculo que tendrían estas lecciones con la sección dedicada a la Psicología Empírica de la Metafísica de Baumgarten — se sabe que Kant usaba esta sección como manual para el dictado del curso de antropología - La autora reconoce este vínculo, pero muestra la importancia que tiene la remisión a otro conjunto de fuentes. Incluso ya en sus primeras lecciones de 1772-1733 Kant refiere su antropología como un Weltkenntnis o Kenntnis des Menschen que en la lección de antropología "Friedländer" de mediados de los años 70 relaciona con un tipo de conocimiento 'pragmático', es decir, en los términos de Kant, a una doctrina de la prudencia. En este contexto, Wilson remite la concepción de lo pragmático en Kant a la tradición que se remonta a Thomasius, pasa por Johann Franz Budde, Andreas Rüdiger entre otros y llega a Christian August Crusius.

Jean-François Goubet propone en "Anthropology-A Legacy from Wolff to Kant?" remitir como una de las fuentes principales de la Antropología en sentido pragmático de Kant el tratamiento de la pneumatología de Wolff, en particular su psicología racional. Aunque, como se remarca al final del artículo, los proyectos de ambos autores son diferentes, 
la referencia a Wolff da cuenta del uso que hace Kant del hombre como animal racional, es decir, como animal que tiene entendimiento (autoconsciencia) y que tiene como destino la razón. Esto se ejemplifica mediante la definición de Wolff de hombre como individuo moral en tanto capaz de preservar memoria de sí mismo, capaz de decir 'yo' y que tiene una dimensión tanto teórica como práctica, la cuestión 'metafísica' del egoísmo, la perfección o perfectibilidad humana y la sabiduría.

"Anthropology from a Logical Point of View: The Role of Inner Sense from Jungius to Kant", de Matteo Favaretti Camposampiero, estudia la antropología como 'antropología del sentido interno' (tal y como Kant define la 'psicología racional' en el $\$ 89$ de la Crítica de la facultad de juzgar) e indaga sobre sus fuentes, entre las cuales se encuentra el 'sentido interior' (o reflexión) de Locke, pero, principalmente, el concepto de 'sentido interno' que se habría desarrollado en la tradición alemana, fundamentalmente, en Wolff y Lambert (pero que se remonta a Descartes, Jungius y Leibniz y tiene resonancias en Baumgarten y Crusius) con independencia del pensamiento de Locke. Lo que le interesa subrayar al autor es el vínculo entre la concepción implicada en esta tradición y su relación con la lógica de los conceptos. Kant habría detectado un error persistente en esta tradición (y que se encuentra todavía presente en el Kant pre-crítico) por el cual se confunde el sentido interno (apercepción empírica), la consciencia de sí en el tiempo, con la apercepción pura, la unidad pura de la conciencia, de la que no podemos tener sensación, sino solo concepto.

Gualtiero Lorini regresa a la cuestión planteada en el primer artículo del libro y pregunta, en "The Rules for Knowing the Human Being: Baumgarten's Presence in Kant's Anthropology", por el alcance de la influencia que pudo tener en su concepción antropológica el manual que usaba Kant en sus clases de antropología, la sección dedicada a la "Psicología Empírica" incluida en la Metaphysica de Baumgarten. A diferencia de Wilson, el autor considera no desdeñable la influencia de este texto para comprender la concepción antropológica de Kant, al menos en las secciones editadas como Didáctica, cuyo tema es el hombre como objeto del sentido interno. El autor muestra que, a diferencia de Wolff, Baumgarten defendía una concepción de la psicología empírica que enfatiza el acceso al alma que tiene la primera persona singular y, por lo tanto, entiende el alma como 'yo' y una concepción de psicología racional en la que se buscaba determinar los 
principios racionales del conocimiento empírico. Estas ideas serían el marco para comprender el aporte antropológico kantiano.

En "Kant on the Vocation and Formation of the Human Being" Ansgar Lyssy desarrolla la inscripción de Kant, todavía no muy comprendida, según el autor, en una de las discusiones centrales de la ilustración alemana, a saber, la discusión en torno a la 'vocación del género humano' [Bestimmung der Menschheit] en la que fueron parte Spalding, Abbt, Mendelssohn, Kant, Schiller, Herder, Fichte y Reinhold. El aporte fundamental de este artículo es mostrar que la concepción histórica de Kant, su concepción del modo en que se desarrollan las diferentes disposiciones humanas a lo largo de la historia, es la respuesta del filósofo a la cuestión ('antropológica') de la vocación del hombre. Como Spalding, Kant incluye dentro de este proyecto no solo el desarrollo de la razón, es decir, la libertad, sino también el desarrollo de las disposiciones animales o sensibles, pero a diferencia de Spalding, la propuesta de Kant apunta a la especie humana en su conjunto y no solo al individuo.

En la Parte II, The Peculiarities of Anthropological Knowledge in Kant: Metaphysics, Morals, Psychology, Politics, Louden, Silva, Tommasi y Sánchez Madrid estudian el impacto que habría tenido la antropología kantiana en el así llamado 'período crítico' y, por lo tanto, los supuestos antropológicos de una diversidad de temas como la moral, la producción artística, la somatología, las enfermedades mentales, etc.

En "The Moral Dimensions of Kant's Anthropology" Robert B. Louden se detiene en la indicación de Kant, en una carta a Marcus Herz de fines de 1773, según la cual la antropología es 'la fuente de todas las ciencias de las costumbres' y, por lo tanto, atiende al uso que Kant le adscribe a la antropología como fundamento de la dimensión práctica, específicamente moral, del ser humano. Contra una fuerte tradición que niega la posibilidad de que una ciencia (empírica) del ser humano pueda fundar un ámbito expresamente trascendental, incluso en tensión con importantes fuentes kantianas, Louden explora esta interesante y novedosa vía, por lo demás, difundida en el marco de la ilustración. El autor ofrece argumentos que indican el sentido en el que la antropología del filósofo es o puede ser considerada una 'antropología moral', mostrando, por ejemplo, cómo en diferentes lugares de su obra Kant reconoce que hay ciertas 'normas-solopara-humanos', es decir, normas 'no puras' que funcionan como imperativos 
categóricos. El conocimiento empírico del ser humano puede devenir 'antropología moral'. El artículo de Louden es valioso no solo por la tesis principal que defiende sino también por su 'estrategia de lectura': su propuesta disputa expresamente una lectura de Kant según la cual ciertas fuentes, pero no otras, definen lo que realmente Kant creyó.

Fernando M. F. Silva, en "Ein Spiel der Sinnlichkeit, durch den Verstand geordnet: Kant's Concept of Poetry and the Anthropological Revolution of Human Imagination", se ocupa de un tema muy desatendido por los estudios kantianos: la concepción kantiana de la poesía [Dichtkunst] - como un juego entre imaginación y entendimiento del que nacen imágenes y representaciones-, de su significado e importancia en las lecciones de antropología. La tesis principal que se defiende en el artículo es que la poesía tiene un rol central en la concepción revolucionaria que Kant tiene de la imaginación humana.

"Somatology: Notes on a Residual Science in Kant and the Seventeenth and Eighteenth Centuries", de Francesco Valerio Tommasi, estudia las menciones de Kant de la 'somatología' y su inscripción en el marco de los desarrollos de esta incipiente disciplina en los siglos XVII y XVIII. Se trata de una ciencia que nace en un contexto en el que la escolástica protestante intentaba suplantar la organización aristotélica de las ciencias. Marco en el cual nacería - este es un aporte muy interesante del artículola ontología, la psicología, la antropología, etc., en el siglo XVI. A diferencia de estas disciplinas, la somatología no tuvo éxito para instituirse como saber disciplinar, consecuencia, según el autor, de la ambigüedad de su objeto: la materia física, en general, pero también el cuerpo animal y el cuerpo humano.

Finalmente, Nuria Sánchez Madrid estudia la concepción kantiana de la enfermedad mental en el marco de su comprensión 'pragmática' de la disciplina antropológica en "Controlling Mental Disorder: Kant's Account of Mental Illness in the Anthropology Writings". La autora muestra cómo la perspectiva de Kant de las dolencias mentales se inscribe, en primer lugar, en la concepción general que Kant tenía de la mente, de la relación entre las facultades y de la exigencia de la unidad de la consciencia, así como también, en segundo lugar, en la concepción que tenía de la antropología como 'pragmática'; como disciplina que considera al hombre como capaz de actuar sobre la naturaleza y, por lo tanto, también sobre sí mismo. Según esta propuesta de lectura la 'terapia' de la enfermedad mental se debería buscar, 
según Kant, en el mismo sitio que le da su origen: así como la pérdida de sentido común coincide con el origen de la enfermedad mental, la interacción social puede prevenirla.

Knowledge, Morals and Practice in Kant's Anthropology es un bello libro del cual pueden sacar gran provecho no solo las personas que investigan la antropología kantiana, sino también aquellas otras que desean tener una comprensión más profunda, renovada y actualizada de la filosofía kantiana. 